\title{
Autonomic Dysfunction in Autism Spectrum Disorder
}

\author{
Andrew P. Owens ${ }^{1 *}$, Christopher J. Mathias ${ }^{2,3,4}$ and Valeria lodice ${ }^{2,3}$ \\ 1 Department of Old Age Psychiatry, King's College London, Institute of Psychiatry, Psychology and Neuroscience, London, \\ United Kingdom, ${ }^{2}$ Autonomic Unit, National Hospital Neurology and Neurosurgery, UCLH NHS Trust, London, \\ United Kingdom, ${ }^{3}$ UCL Queen Square Institute of Neurology, University College London, London, United Kingdom, \\ ${ }^{4}$ Neurovascular Medicine Unit, Lindo Wing, St Mary's Hospital, Imperial College NHS Healthcare Trust, London, \\ United Kingdom
}

\section{OPEN ACCESS}

Edited by:

Darren J. Edwards, Swansea University, United Kingdom

Reviewed by:

Lynn Waterhouse, The College of New Jersey,

United States

Britta Berglund,

Retired, Tumba, Sweden

*Correspondence: Andrew P. Owens andrew.owens@kcl.ac.uk

Received: 30 September 2021 Accepted: 29 November 2021 Published: 30 December 2021

Citation:

Owens AP, Mathias CJ and lodice V (2021) Autonomic Dysfunction in Autism Spectrum

Disorder.

Front. Integr. Neurosci. 15:787037. doi: 10.3389/fnint.2021.787037
Background: There have been previous reports of enhanced sympathoexcitation in autism spectrum disorder (ASD). However, there has been no formal investigation of autonomic dysfunction in ASD. Also, the joint hypermobile form of EhlersDanlos syndrome (hE-DS) that maybe overrepresented in ASD and orthostatic related autonomic dysfunction. This study examined the comorbidity of ASD, autonomic dysfunction and hE-DS in two UK autonomic national referral centers. Proven, documented and globally accepted clinical autonomic investigations were used to assess neuro-cardiovascular autonomic function in a cohort of ASD subjects and in age-matched healthy controls.

Methods: Clinical data from 28 referrals with a confirmed diagnosis of ASD over a 10year period were compared with 19 age-matched healthy controls. Autonomic function was determined using methods established in the centers previously described in detail.

Results: 20/28 ASD had a diagnosed autonomic condition; 9 had the postural tachycardia syndrome (PoTS), 4 PoTS and vasovagal syncope (WS), 3 experienced presyncope, 1 essential hyperhidrosis, 1 orthostatic hypotension, 1 WS alone and 1 a combination of PoTS, WS and essential hyperhidrosis. 16/20 ASD with autonomic dysfunction had hE-DS. In ASD, basal heart rate and responses to orthostatic tests of autonomic function were elevated, supporting previous findings of increased sympathoexcitation. However, sympathetic vasoconstriction was impaired in ASD.

Conclusion: Intermittent neuro-cardiovascular autonomic dysfunction affecting heart rate and blood pressure was over-represented in ASD. There is a strong association with hE-DS. Autonomic dysfunction may further impair quality of life in ASD, particularly in those unable to adequately express their experience of autonomic symptoms.

Keywords: autism, autonomic nervous system, postural tachycardia syndrome, syncope, hyperhidriosis, hypermobile ehlers danlos syndrome, dysautonomia

\section{INTRODUCTION}

Homeostatic regulation is facilitated by the autonomic nervous system (ANS) and its ability to mediate activity of bodily organs especially the heart and blood vessels via peripheral efferent nerves. The term "autonomic" derives from the fact that its function is largely beyond conscious control. The ANS has sympathetic nervous system (SNS) and the parasympathetic nervous 
system (PNS) efferent pathways. The SNS increases effector organ activity predominantly via the catecholamines, such as noradrenaline (NA) at the neuro-effector junction. The PNS promotes downregulation of effector organ activity, through neuro-transmitters such as acetylcholine, slowing heart rate and facilitating food digestion by its effects on the gastrointestinal tract.

The literature investigating autonomic function in autism spectrum disorder (ASD), particularly during behavioral studies, report a prevalence of enhanced sympathoexcitation (increased cardiovascular and sudomotor activity) (Althaus et al., 2004; Bal et al., 2010; Porges et al., 2013). Autonomic abnormalities have also been reported in relation to psychological factors using self-report measures (Ming et al., 2011) by those with ASD. However, studies into pupillary responses and cardiovagal function have produced contradictory findings. Some studies have found smaller pupil size in ASD (Martineau et al., 2011), unlike others with increased pupil size (Anderson et al., 2013). In comparison to controls and on cardiovagal function, some studies have found vagally-mediated cardiac modulation to be increased in ASD (Toichi and Kamio, 2003), whilst others have found the opposite (Bal et al., 2010). Autonomic dysregulation has been proposed by some as a contributing factor to the neuropsychiatric symptoms in ASD (Hutt et al., 1964; Porges et al., 1996, 2013). However, no study has yet investigated autonomic function or dysfunction (dysautonomia) in ASD. The purpose of this study was to use clinical laboratory investigations to determine if there was autonomic dysfunction or an autonomic disorder in a cohort with ASD, in comparison with age-matched healthy controls.

Autonomic dysfunction can present as a primary or secondary feature, complicating neurological, cardiovascular, endocrine, metabolic and genetic disorders. There are:

- "fixed" forms, with autonomic damage and failure that usually is irreversible (such as spinal cord injury), and potentially progressive (as in Parkinson's disease with autonomic failure and multiple system atrophy) (Bannister and Mathias, 2013), the latter usually occurring in middleto-late adulthood;

- "intermittent" forms, with no obvious damage to autonomic nerves, but a transient dysregulation of otherwise normal autonomic function due to a specific allostatic stimulus, such as standing upright as in the postural tachycardia syndrome (PoTS) or with temperature change (thermoregulatory dysfunction). Intermittent autonomic disorders can present during adolescence and early adulthood, often with a positive family history (Mathias et al., 1998, 2012; Kaufmann et al., 2003).

Fixed forms of autonomic disorders with autonomic failure are characteristically due to trauma to the ANS or neurodegeneration (spinal cord injury or Parkinsonism), and thus intermittent autonomic dysfunction is more likely to occur in ASD. A common consequence of orthostatic intolerance is fainting (syncope). Autonomic (neurally) mediated syncope (AMS) usually occurs due to a transient breakdown of homeostatic autonomic reflex arcs during standing and may be due to:

- Situational syncope-attributable to a specific stimulus, such as straining during weightlifting or playing a wind instrument, often increasing intrathoracic pressure;

- Vasovagal syncope (VVS) - the most common (80\% of cases) form of syncope. In VVS, sympathoexcitation (increased heart rate and sweating) can precede the drop in blood pressure due to vasodilatation (due to sympathetic withdrawal) and bradycardia (due to increased vagal/parasympathoexcitation);

- Carotid sinus sensitivity-typically in those aged $>50$ years from aberrant baroreceptor overactivation in nerves around the carotid artery.

The most common form of orthostatic intolerance probably is PoTS, defined by a heart rate increase of $>30$ BPM or heart rate of $>120 \mathrm{BPM}$ with symptoms of orthostatic intolerance (dizziness, palpitations), but without orthostatic hypotension (Owens et al., 2017) when standing upright or within $10 \mathrm{~min}$ of orthostasis or head-up tilt (HUT) (Freeman et al., 2011). Some consider PoTS under hyperadrenergic or neuropathic phenotypes (Benarroch, 2012), although this often is not readily determined on clinical evaluation or testing. Infection, deconditioning, hypovolemia and impaired cerebral autoregulation have been implicated as triggers in PoTS or its pathophysiology.

Essential hyperhidrosis is the most common form of intermittent autonomic thermodysregulation and is defined by profuse and uncontrollable sweating exceeding that needed to maintain thermostasis, often causing significant functional impairment, and without a definitive cause. Essential hyperhidrosis can present as excessively local, on the palms, soles and axillae, or can be generalized. The prevalence of essential hyperhidrosis has been estimated as $2-3 \%$ (Ocon et al., 2009; Lai et al., 2015)but the etiology remains uncertain. Once a secondary cause of hyperhidrosis has been eliminated (e.g., drug side-effect), sudomotor activity can be measured by a thermoregulatory sweat test (Moraites et al., 2014).

Orthostatic intolerance is often associated with the joint hypermobility syndrome and Ehlers-Danlos syndrome (E-DS) Type III, now considered as the joint hypermobile form of E-DS (hE-DS). It is a heritable rheumatological condition characterized with variant collagen distribution across connective tissue, but without an identified gene as yet. hE-DS appears overrepresented in ASD (Eccles et al., 2014) and may predispose to orthostatic intolerance and PoTS (Mathias et al., 2012; Eccles et al., 2015). Despite the co-occurrence of hE-DS in ASD and forms of orthostatic intolerance, a detailed investigation into the presence of autonomic disorders in ASD has not yet been undertaken. Autonomic dysfunction in those with ASD may further impair morbidity and quality of life (QoL), and this study examined the potential comorbidity of ASD and clinical autonomic dysfunction, focusing also on intermittent autonomic disorders, in two UK national referral centers for autonomic disorders that have worked in unison (the Autonomic Unit at the 
National Hospital for Neurology and Neurosurgery, University College London Hospitals, Queen Square, and the Pickering Unit at St Mary's Hospital, Imperial College Healthcare NHS Trust). There also was an emphasis on clinical features that may further aid our understanding of autonomic function studied in detail in ASD, which previously has not been addressed.

\section{MATERIALS AND METHODS}

\section{Ethics}

National (London-Harrow Research Ethics Committee) and institutional (University College London and Imperial College London) ethics were acquired for the examination of the medical records of all patients referred over a 10-year period (2007-2017) with a confirmed diagnosis of ASD.

\section{Participants}

We identified 28 autonomic evaluation referrals with a confirmed diagnosis of ASD (aged $29 \pm 8.66,12$ female, 18 male). ASD data was compared to existing age-matched healthy control data ( $n=19$, mean age $29 \pm 12.85,10$ female, 9 male). None of the healthy control group had a history of cranial injury, neurological disorder, cognitive impairment or learning disability. The controls were recruited from Imperial College London, University College London and members of the local community. All participants provided written informed consent.

\section{Medication}

Medication of relevance to autonomic conditions in those with ASD is detailed in Table $\mathbf{1}$ of the results section.

\section{Autonomic Assessment}

Heart rate and the electrocardiogram (ECG) were continually monitored online (PowerLab 16/30/ECG (Bioamp) (AD Instruments, Oxford, United Kingdom) and analyzed offline (Labchart 7). Blood pressure was continually recorded using digital photoplethysmography (Finometer, Smart Medical, Gloucestershire, United Kingdom) and heart rate and blood pressure measures were taken using automated sphygmomanometry (Dinamap Pro400V2, GE Healthcare, Buckinghamshire, United Kingdom). The evaluations were performed during the tests outlined, and performed in the laboratories (with identical equipment) in the two autonomic laboratories that were an integral part of the national referral centers.

Before autonomic testing, each patient underwent detailed clinical evaluation (history/examination) by a consultant autonomic physician who documented relevant information, to include family history of orthostatic intolerance. This included relevant autonomic symptoms (such as postural dizziness and palpitations) and factors worsening intolerance (such as standing still, food, exertion, heat and in female the menstrua; period). Included was assessment for hE-DS, based on clinical examination and current criteria (Castori et al., 2017). Historical or current psychiatric conditions diagnosed by a mental healthcare professional were recorded. Informed
TABLE 1 | Overview of ASD referrals with a diagnosable form of autonomic dysfunction.

\begin{tabular}{|c|c|c|}
\hline Autonomic diagnosis & $\begin{array}{l}\text { Hypermobile form of } \\
\text { Ehlers-danlos } \\
\text { syndrome (hE-DS) }\end{array}$ & Medication \\
\hline $\begin{array}{l}9 \text { PoTS ( } 3 \text { female, } 6 \\
\text { male) }\end{array}$ & 7/9 (78\%) & 8 analgesic \\
\hline $\begin{array}{l}4 \text { Comorbid PoTS and } \\
\text { WS ( } 4 \text { female) }\end{array}$ & $4 / 4$ (100\%) & 7 antidepressant \\
\hline $\begin{array}{l}3 \text { Presyncope ( } 2 \\
\text { female, } 1 \text { male) }\end{array}$ & $3 / 3(100 \%)$ & 5 anti-epileptic drug \\
\hline 1 WS (male) & $1 / 1(100 \%)$ & $\begin{array}{l}4 \text { proton pump inhibitor } \\
4 \text { anti-inflammatory } 4 \\
\text { antipsychotic }\end{array}$ \\
\hline $\begin{array}{l}1 \text { Generalized EH } \\
\text { (female) }\end{array}$ & - & $\begin{array}{l}3 \text { Salbutamol inhaler } 3 \\
\text { migraine treatment } 3 \\
\text { anti-nausea }\end{array}$ \\
\hline $\begin{array}{l}1 \text { orthostatic } \\
\text { hypotension (female) }\end{array}$ & $1 / 1(100 \%)$ & $\begin{array}{l}2 \text { anti-bladder } \\
\text { spasmodic } 2 \\
\text { constipation medication } \\
2 \text { antihistaminics }\end{array}$ \\
\hline $\begin{array}{l}1 \text { Comorbid PoTS, } \\
\text { WS, and craniofacial } \\
\text { EH (male) }\end{array}$ & - & Medication \\
\hline 20/25 (80\%) & $16 / 20(80 \%)$ & \\
\hline
\end{tabular}

EH, essential hyperhidrosis; POTS, postural tachycardia syndrome; WS, vasovagal syncope. The numbers and percentage with an autonomic diagnosis and hE-DS are indicated below.

consent was received from each patient prior to testing and, if tolerable, patients were asked to discontinue any medications that may affect autonomic function $24-48 \mathrm{~h}$ prior to testing and abstain from caffeine, nicotine and vigorous exercise $24 \mathrm{~h}$ before testing. Established and validated clinical protocols (Bannister and Mathias, 2013; Mathias et al., 2013) assessed:

- Sympathetic vasoconstriction during mental arithmetic (1 min), isometric exercise $(3 \mathrm{~min}$ ) and cutaneous cold pressor responses (90 s). Pressor maneuvers, including isometric (hand-grip) exercise, cutaneous cold application, deep breathing and mental arithmetic provide an index of sympathetic nerve activity (SNA) and induce autonomic cardiovascular changes, particularly in blood pressure, which is regulated via the SNS. Isometric and cutaneous cold pressor stimuli raise blood pressure via activation of sympathetic efferent nerve pathways and provide more responsive data in comparison to mental arithmetic or other pressor tests. Peripheral receptors are activated but in both cutaneous cold or isometric exercise tests there is an important central command (isometric) or nociceptive (cold) role, which is more pronounced in isometric exercise leading to a greater increase in SNA compared to the cold pressor test;

- Cardiac vagal function during respiratory sinus arrhythmia (RSA, $1 \mathrm{~min}$ ). In normal subjects there is an increase in heat rate during inspiration and a decrease in HR during expiration, known as "respiratory sinus arrhythmia" (RSA), which is a measure of the functional endpoint of 
TABLE 2 | Summary of autonomic test data in controls and ASD.

\begin{tabular}{|c|c|c|c|c|c|c|}
\hline Groups & Autonomic domain & Baseline & Isometric exercise & Cold pressor & Mental arithmetic & Head up tilt \\
\hline \multirow[t]{3}{*}{ Healthy controls } & Heart rate (BPM) & $63 \pm 8.49$ & $77 \pm 10.24$ & $61 \pm 8.97$ & $62 \pm 8.11$ & $78 \pm 8.99$ \\
\hline & $\mathrm{SBP}(\mathrm{mmHg})$ & $122 \pm 12.49$ & $146 \pm 13.26$ & $133 \pm 17.35$ & $118 \pm 10.62$ & $116 \pm 16.51$ \\
\hline & $\mathrm{DBP}(\mathrm{mmHg})$ & $66 \pm 5.84$ & $88 \pm 9.93$ & $61 \pm 8.97$ & $65 \pm 9.08$ & $69 \pm 10.31$ \\
\hline \multirow[t]{3}{*}{ Autism spectrum disorder } & Heart rate (BPM) & $77 \pm 14.66^{\star}$ & $87 \pm 17.27^{\star}$ & $79 \pm 16.52^{*}$ & $81 \pm 13.07^{\star}$ & $99 \pm 16.46^{*}$ \\
\hline & $\mathrm{SBP}(\mathrm{mmHg})$ & $118 \pm 11.02$ & $132 \pm 45^{\star}$ & $126 \pm 10.72^{\star}$ & $118 \pm 10.55^{\star}$ & $118 \pm 12.5^{\star}$ \\
\hline & $\mathrm{DBP}(\mathrm{mmHg})$ & $69 \pm 8.37$ & $78 \pm 7.17^{\star}$ & $75 \pm 7.12$ & $70 \pm 9.48$ & $74 \pm 8.11$ \\
\hline
\end{tabular}

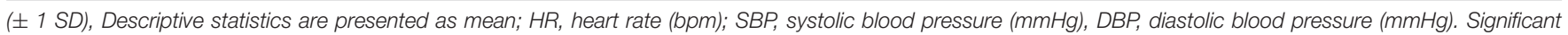
difference in ASD from controls indicated by*. The bold values refer to significant findings.

cardioinhibitory vagal fibers emanating from the nucleus ambiguus in the brainstem. In this study cardiovagal function was assessed by subtracting the minimum heart rate response from the maximum heart rate response to deep breathing averaged over a minimum of 5 breathing cycles (HRDB) (Bannister and Mathias, 2013; Mathias et al., 2013);

- Baroreflex cardiovagal and adrenergic function using the Valsalva maneuver, measures central and peripheral baroreflex pathways. The Valsalva maneuver involves forced expiratory pressure $(40 \mathrm{mmHg})$ against a blocked airway for $10-15 \mathrm{~s}$ (repeated $2 / 3$ times). The increase in intrathoracic pressure causes heart rate and blood pressure changes that can be used to calculate indices of sympathetic and parasympathetic activity;

- Orthostatic tolerance, baroreflex and adrenergic function during head-up tilt (HUT, 9 min). HUT is used to diagnose various forms of orthostatic intolerance. In healthy subjects the initial blood pressure fall induced by HUT should recover within $60 \mathrm{~s}$ as decreased venous return to the heart causes reduced stroke volume and cardiac output, activating arterial baroreceptors and cardiopulmonary mechanoreceptors that signal autonomic brain centers to increase sympathetic nerve activity, raising $\mathrm{HR}$ and causing vasoconstriction in various vascular beds to compensate for gravitational demands. In normal subjects in whom the baroreflex is intact, HUT of $45-90^{\circ}$ should not provoke a prolonged fall in BP. HUT is a valuable investigation that can be terminated if syncopal, pre-syncopal or other OI-related symptoms are recorded or reported by the participant.

During orthostatic challenge, lower extremities were examined for signs of vascular pooling, such as mottling and cyanotic discoloration, indicative of impaired venous return.

\section{Statistical Analysis}

Statistical analysis was performed using SPSS (version 25). Descriptive statistics are presented as mean ( $\pm 1 \mathrm{SD}$ ) for normally distributed data. Data were tested (Shapiro-Wilk test) for normality and homogeneity of variance. Quantitative variables were compared at single time points by independent $t$-tests for two groups to compare the results of clinical autonomic testing in patients with ASD and age-matched healthy controls. When appropriate, non-parametric tests were used to compare groups

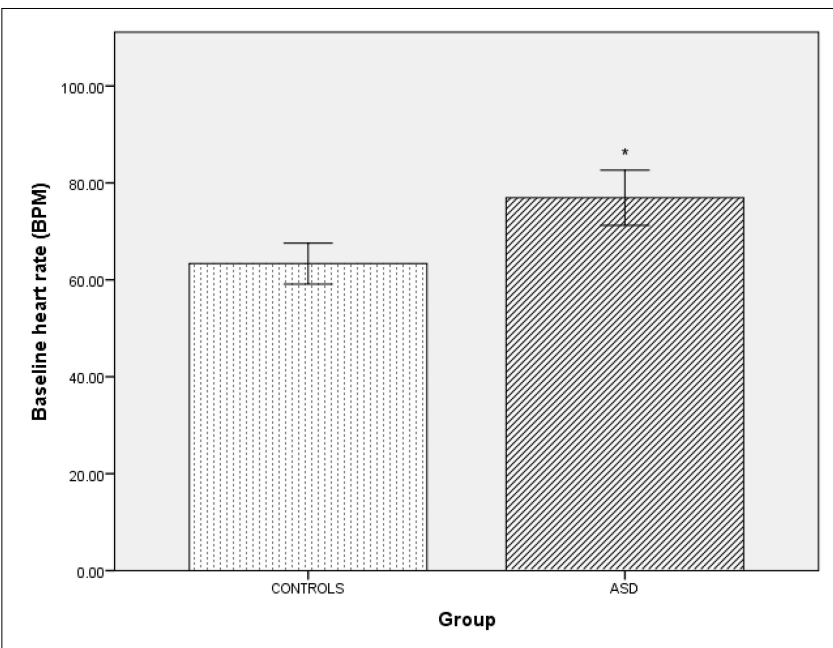

FIGURE 1 || Baseline supine heart rate in controls and ASD. BPM, beats per minute. *Refer to a statistically significant finding.

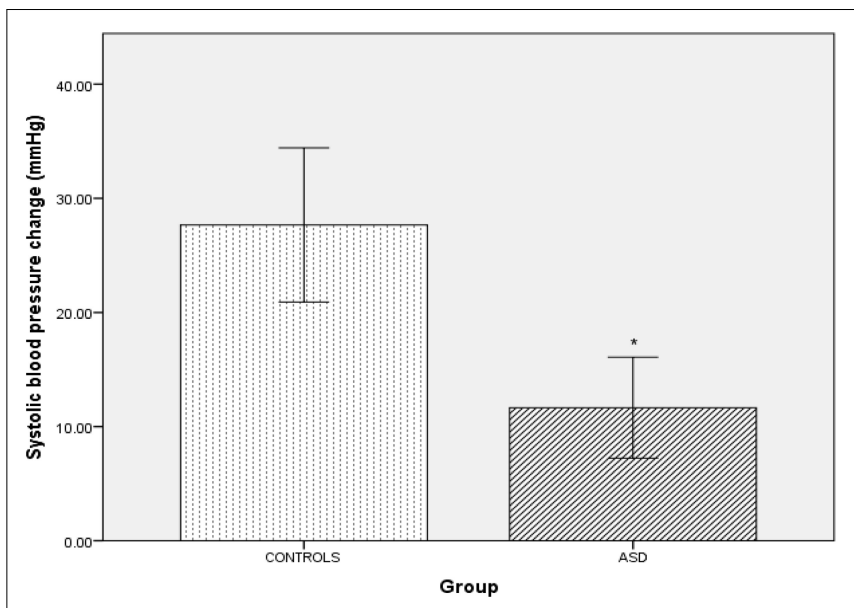

FIGURE 2 | Changes in mean systolic blood pressure from baseline during isometric exercise in controls and ASD. $\mathrm{mmHg}$, millimeters of mercury. *Refer to a statistically significant finding.

(Mann-Whitney $U$ Test). Statistical significance was specified as a 2 -tailed $p$-value of $<0.05$. 


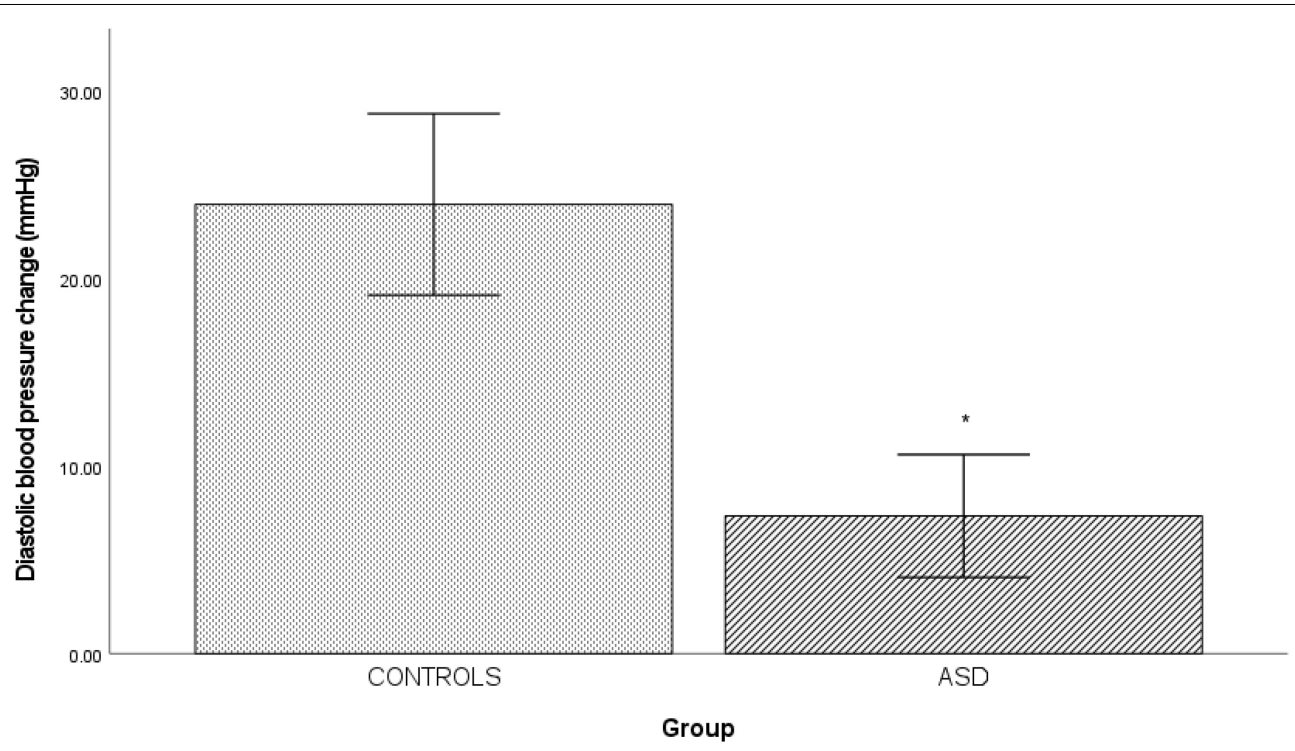

FIGURE 3 | Changes in mean diastolic blood pressure from baseline during isometric exercise in controls and ASD. mmHg, millimeters of mercury. *Refer to a statistically significant finding.

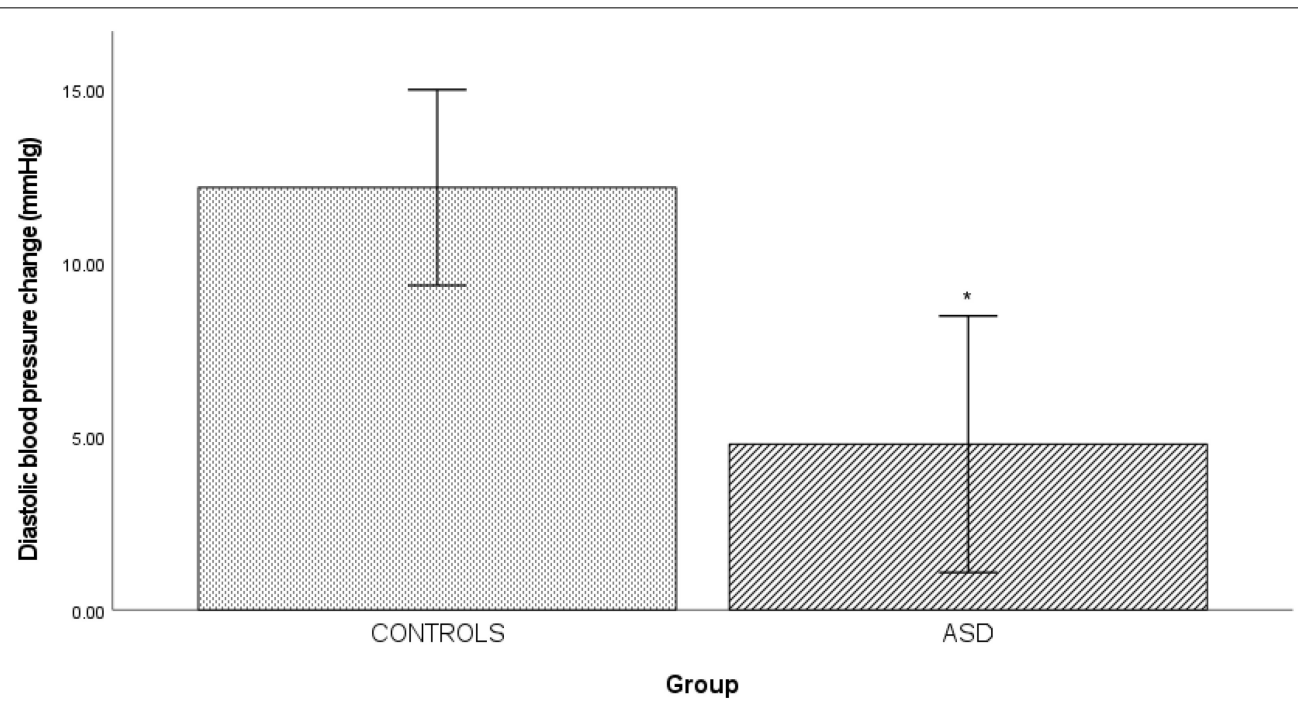

FIGURE 4 | Changes in mean diastolic blood pressure from baseline during cold pressor in controls and ASD. mmHg, millimeters of mercury. ${ }^{\star}$ Refer to a statistically significant finding.

\section{RESULTS}

\section{Clinical Data}

Of the 28 ASD group, 3 were unable to complete the full test protocol due to anxiety, joint pain or apneic episodes but their data was analyzed until the cessation of testing. Group mean values for each autonomic test are shown in Table 2.

\section{Baseline}

The ASD group had a significantly $[t(42)=-4.69, p=0.001]$ higher supine resting heart rate $(77 \mathrm{bpm} \pm 14.66)$ than the control group (63 bpm \pm 8.49 , Figure 1). Between-group blood pressure measures were comparable.

\section{Isometric Exercise}

Pressor stimuli, such as isometric exercise, cutaneous cold application and mental arithmetic, examine SNS vasoconstriction. In the ASD group, there was a significantly $[t(36)=4.25, p=<0.001)$ blunted $(\Delta 11 \mathrm{mmHg} \pm 9.44)$ systolic blood pressure response to isometric exercise compared to the control group $(\Delta 15 \mathrm{mmHg} \pm 13.57$, Figure 2$)$.

In the ASD group, there was a significantly $[t(37)=6.12$, $p=0.001]$ blunted diastolic blood pressure response $(\Delta$ 
$7.33 \mathrm{mmHg} \pm 7.19)$ to isometric exercise compared to the control group $(\Delta 23.44 \mathrm{mmHg} \pm 9.73$, Figure 3$)$.

\section{Cold Pressor Test}

In the ASD group, there was a significantly $[t(38)=3.20$, $p=0.003$ ] blunted diastolic blood pressure response $(\Delta$ $4.77 \mathrm{mmHg} \pm 8.34)$ to isometric exercise compared to the control group $(\Delta 12.17 \mathrm{mmHg} \pm 5.67$, Figure 4$)$.

\section{Mental Arithmetic}

In the ASD group there was a significantly $[t(33)=0.333$, $p=0.014]$ blunted diastolic blood pressure response $(\Delta$ $2.26 \mathrm{mmHg} \pm 5.23)$ to mental arithmetic compared to the control group (Figure 5).

\section{Head-Up Tilt}

In the ASD group during head-up tilt, there was a significant $(t(41)=-2.32, p=0.025)$ increase in heart rate $(\Delta 22 \mathrm{bpm} \pm$ $10.60)$ compared to the control group $(\Delta 15 \mathrm{bpm} \pm 8.24$, Figure 6).

\section{Respiratory Sinus Arrythmia}

Cardiovagal function was assessed by subtracting the minimum heart rate response from the maximum heart rate response to deep breathing averaged over a minimum of 5 breathing cycles. During paced deep breathing, respiratory sinus arrhythmia indicated significantly $[t(30)=-3.60, p=0.001]$ increased cardiac acceleration during inspiration in the ASD group (93 bpm + 13.59) compared to the healthy control group (72 bpm \pm 12.27 ). Vagally-mediated cardiac deceleration during expiration in the ASD group was significantly $[t(30)=-3.58$, $p=0.001]$ attenuated $(75 \mathrm{bpm} \pm 14.31)$ compared to the healthy control group (55 bpm \pm 10.07$)$.

\section{Valsalva Maneuver}

Four ASD subjects had a normal variant flat top BP response (lack of arterial pressure decrease during phase II early) to Valsalva maneuvers. One patient was unable to follow the guidance required to perform the Valsalva maneuver and was therefore excluded from analysis. During phase II early of the Valsalva maneuver, the typical fall in blood pressure due to reduced venous return and stroke volume was significantly $[t(19)=-2.23$, $p=0.037]$ attenuated in the ASD group $(\Delta 36 \mathrm{mmHg} \pm 15.42)$ compared to the control group $(\Delta 19 \mathrm{mmHg} \pm 1$, Figure 7$)$.

\section{Autonomic Diagnoses}

20/28 (71\%) ASD subjects had a recognizable autonomic disorder (Table 1). 9 ASD had PoTS, 4 had PoTS and VVS, 3 experienced presyncope (i.e., testing was aborted as the blood pressure and heart rate profiles indicated they were about to faint), 1 had VVS, 1 had (generalized) essential hyperhidrosis, 1 had orthostatic hypotension and 1 had comorbid PoTS, VVS and essential (predominantly craniofacial) hyperhidrosis. Of the 16/20 (80\%) ASD patients with an autonomic disorder also had hE-DS.

\section{Psychiatric Co-morbidities}

These are listed, with diagnosis and the numbers affected. One had a pre-existing diagnosis of severe cognitive impairment, 1 a pre-existing diagnosis of learning difficulties, 1 a preexisting diagnosis of anxiety disorder, 1 a pre-existing diagnosis of obsessive-compulsive disorder, self-harm, 1 a pre-existing diagnosis of Tourette's syndrome, 1 a pre-existing diagnosis of

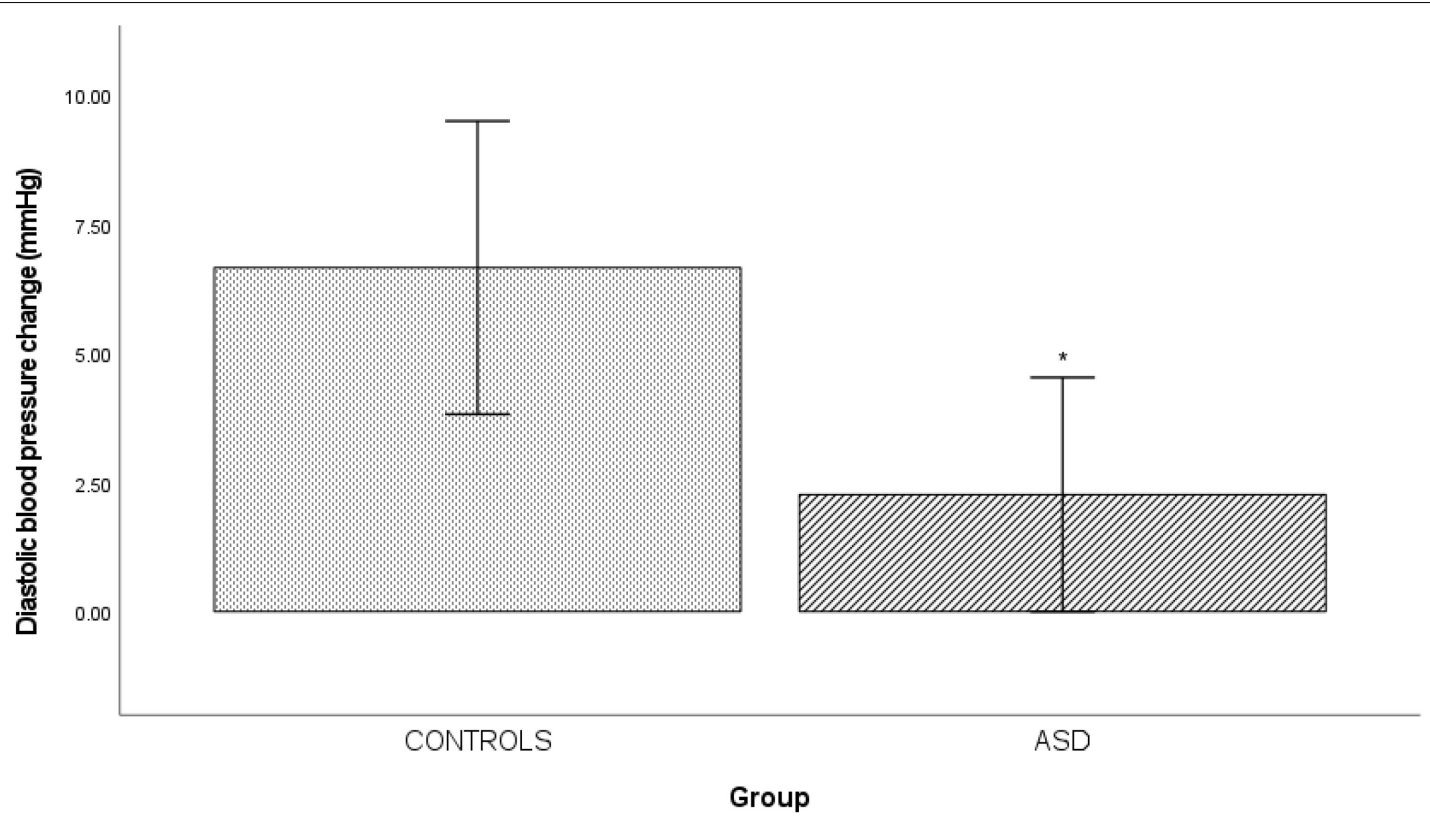

FIGURE 5 | Changes in mean diastolic blood pressure from baseline during mental arithmetic in controls and ASD. mmHg, millimeters of mercury. * Refer to a statistically significant finding. 


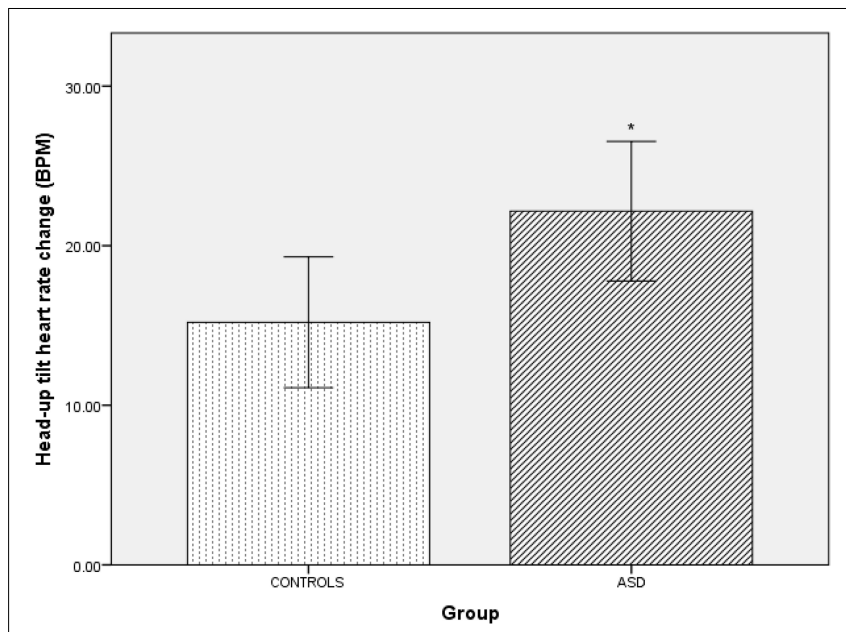

FIGURE 6 | Changes in heart rate from baseline during head-up tilt in controls and ASD. BPM, beats per minute. *Refer to a statistically significant finding.

schizophrenia, 1 a pre-existing diagnosis of depression and 2 with a pre-existing diagnosis of anxiety disorder.

\section{DISCUSSION}

This is the first study of detailed cardiovascular and thermoregulatory autonomic testing in ASD in two national referral centers for autonomic disorders. The association with hE-DS being over-represented in ASD and orthostatic intolerance also was determined. In ASD, 20/25 (80\%) had an autonomic condition; 9 had PoTS, 4 had PoTS and VVS, 3 experienced presyncope (i.e., testing aborted due to the beat-tobeat blood pressure and heart rate indicating they were about to faint) during standing or HUT, 1 had VVS, 1 had generalized essential hyperhidrosis, 1 had orthostatic hypotension and 1 had PoTS, VVS and essential (craniofacial) hyperhidrosis. $80 \%$ of ASD with dysautonomia had hE-DS.

Resting heart rate was significantly higher in ASD, with a greater increase during HUT, favoring enhanced sympathoexcitation. During phase II early of the Valsalva maneuver, the fall in blood pressure was significantly reduced in ASD compared to controls. This differs from the typical Valsalva profile in PoTS (Aman, 2004) with a trend toward flat top Valsalva profiles and complements the probably increased sympathetic tone during baseline. PoTS subjects also may have hypovolemia, which could reduce cardiac output. Whether this may occur in is unclear.

Pressor responses were blunted in ASD, unlike increased cardiac sympathoexcitation during HUT in this group, and this is of relevance to $\beta$-adrenergic antagonists that are reported to reduce some of the negative common behaviors, such as aggression and impulsiveness in ASD (Boddaert et al., 2004). The attenuated diastolic blood pressure (representing peripheral resistance) responses to isometric exercise, cold pressor and mental arithmetic in ASD also may relate to the prevalence of hE-DS, where there may be greater distensibility

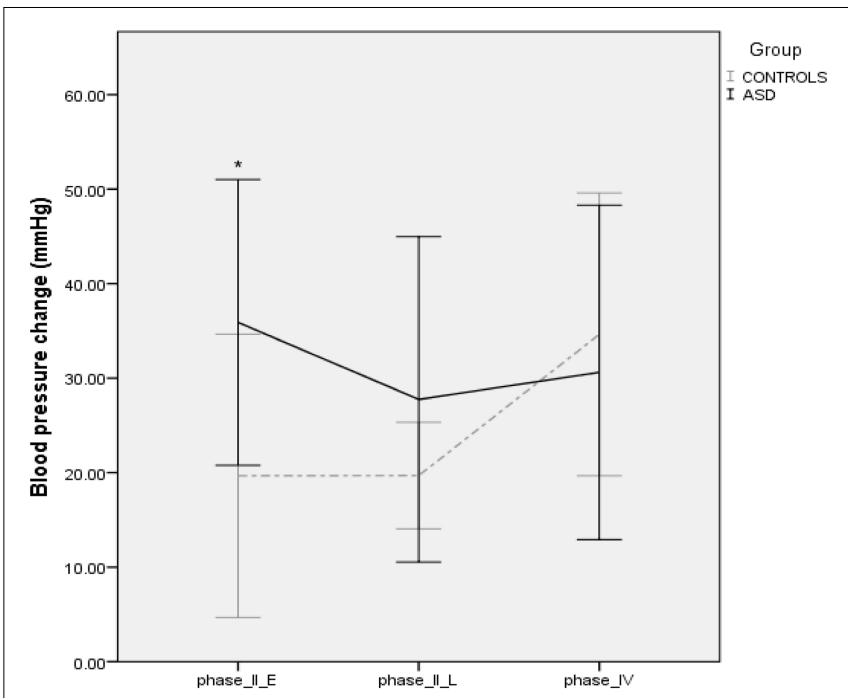

FIGURE 7 | Blood pressure profiles during the Valsalva maneuver in controls and ASD. mmHg, millimeters of mercury. *Refer to a statistically significant finding.

of blood vessels (with the collagen deficit), thus diminishing vasoconstriction during pressor tests, and predisposing to orthostatic intolerance.

Neuroimaging studies have offered insights into potential central reasons for perturbed autonomic function in ASD. Both ASD and hE-DS are associated with reductions in right superior temporal volume, an area vital for the sensory processing of social emotional information (Boddaert et al., 2004), and in amygdala development (Eccles et al., 2012; Bernhardt et al., 2016). The amygdala is a key structure in the central autonomic network and its activity, along with the dorsal anterior cingulate cortex, predicts changes in heart rate (Jänig and Häbler, 2003) and cardiac contractility during stress (Dalton et al., 2005). Moreover, in ASD, altered functional connectivity and activation (Hadjikhani et al., 2009; Ebisch et al., 2011; Watanabe et al., 2012; Duerden et al., 2013) during emotional processing of the insular, another part of the central autonomic network (Bennaroch, 1993), has been reported. The anterior insular cortex (AIC) contains a significant number of "von Economo neurons," which are large bipolar, spindle-shaped projection neurons (Seeley et al., 2012). These neurons are prevalent in humans and are mainly situated in layer $\mathrm{Vb}$ of the anterior cingulate cortex and the fronto-insular cortex (i.e., the junction of AIC and posterior orbito-frontal cortex) and are specifically associated with efferent autonomic mediation to the brainstem and spinal cord (Evrard, 2019) and afferent visceral feedback (known as, "interoception") (Allman et al., 2011). In comparison to controls, ASD have von Economo abnormalities, morphological differences (Simms et al., 2009) and a significantly greater ratio of von Economo neurons to pyramidal neurons (Santos et al., 2011), of probable relevance to autonomic disturbances reported here, and interoceptive sensitivity reported previously in ASD (Quattrocki and Friston, 2014). 
The psychiatric comorbidities in some with ASD are likely to relate to ASD itself rather than the anxiety symptoms reported in PoTS, VVS and essential hyperhidrosis, which are typically sub-clinical and specific to somatic hypervigilance (Eccles et al., 2015; Owens et al., 2016, 2017). Two ASD subjects, one with PoTS and another with generalized hyperhidrosis were cognitively impaired and their autonomic symptoms were pronounced enough to be noted by their caregivers, hence their referral for autonomic investigation. This may be of relevance to some of their symptoms and needs consideration in their overall management. The co-occurrence of ASD and hEDS has only fairly recently (since 2014) (Shetreat-Klein et al., 2014) been formally reported in a well-characterized dataset. Overlapping symptoms include, anxiety, pain, sleep disruption and gastrointestinal problems (Tinkle et al., 2017). And this study builds on our previous findings investigating the prevalence of neurodevelopmental disorders in those with autonomic dysfunction and hEDS (Sandroni et al., 2000). The current findings draw together the previously separate investigations of ASD and hE-DS with those exploring ASD and autonomic function, and indicates these two currently separate bodies of work could be drawn together to elucidate the overlap and interplay between ASD and autonomic dysfunction, which should benefit diagnostic evaluation and treatment strategies.

This retrospective study was in a relatively small sample of ASD and studies in larger numbers are required to provide further in-depth analyses of ANS function/dysfunction in ASD. The current data also was collected from two national referral centers for autonomic conditions, with subjects referred because of possible autonomic dysfunction. Further studies in a larger cohort with ASD, regardless of autonomic symptoms is needed. There are clear differences between ASD and controls, with 16/20 (80\%) of ASD having a confirmed autonomic condition, also with hEDS suggesting that further examination of the overlapping relationship between ASD, autonomic dysfunction and hEDS may be informative, particularly in relation to biomarker identification. Additional studies with larger numbers, a broader spectrum of autonomic assessments and detailed autonomic evaluation should provide further in-depth analysis of ANS function and dysfunction in ASD, and the relationship to their key issues. Studies in ASD that are apparently asymptomatic with autonomic dysfunction are likely to provide further information.

\section{REFERENCES}

Allman, J. M., Tetreault, N. A., Hakeem, A. Y., Manaye, K. F., Semendeferi, K., Erwin, J. M., et al. (2011). The von Economo neurons in the frontoinsular and anterior cingulate cortex. Ann. NY Acad. Sci. 214, 495-517. doi: 10.1111/j.17496632.2011.06011.x

Althaus, M., Van Roon, A. M., Mulder, L. J., Mulder, G., Aarnoudse, C. C., and Minderaa, R. B. (2004). Autonomic response patterns observed during the performance of an attention-demanding task in two groups of children with

\section{CONCLUSION}

In this study elevated heart rate while supine and upright was present in ASD in comparison to controls, suggestive of increased sympathoexcitation. Sympathetic vasoconstriction, however, appeared impaired in ASD. Intermittent autonomic dysfunction (cardiovascular and thermoregulatory) was overrepresented in ASD, and there was a strong association with hE-DS. Abnormalities in the central autonomic network may be contributory and further compromise autonomic function. Autonomic dysfunction may impair quality of life in ASD, particularly in those unable to express their experience of such symptoms. The interaction of autonomic symptoms with cognitive-affective and socio-emotional symptomatology warrants further investigation in larger groups with ASD, including those initially with no apparent autonomic features.

\section{DATA AVAILABILITY STATEMENT}

The raw data supporting the conclusions of this article will be made available by the authors, without undue reservation.

\section{ETHICS STATEMENT}

The studies involving human participants were reviewed and approved by London-Harrow Research Ethics Committee. The patients/participants provided their written informed consent to participate in this study.

\section{AUTHOR CONTRIBUTIONS}

$\mathrm{AO}, \mathrm{CM}$, and VI: conception and design of study, analysis, interpretation of data, and drafting and revising manuscript. AO: acquisition of data. All authors contributed to the article and approved the submitted version.

\section{FUNDING}

AO was funded by a research fellowship from the Autonomic Charitable Trust. The funders had no role or influence in study design, data collection, analysis and interpretation of data, writing the report or the decision to submit the manuscript for publication.

autistic-type difficulties in social adjustment. Psychophysiology 41, 893-904. doi: 10.1111/j.1469-8986.2004.00252.x

Aman, M. G. (2004). Management of hyperactivity and other acting-out problems in patients with autism spectrum disorder. Semin. Pediatric Neurol. 11, 225-228. doi: 10.1016/j.spen.2004.0 7.006

Anderson, C. J., Colombo, J., and Unruh, K. E. (2013). Pupil and salivary indicators of autonomic dysfunction in autism spectrum disorder. Dev. Psychobiol. 55, 465-482. doi: 10.1002/dev.21051 
Bal, E., Harden, E., Lamb, D., Van Hecke, A. V., Denver, J. W., and Porges, S. W. (2010). Emotion recognition in children with autism spectrum disorders: relations to eye gaze and autonomic state. J. Autism Dev. Disord. 40, 358-370. doi: 10.1007/s10803-009-0884-3

Bannister, R., and Mathias, C. J. (2013). "Introduction and classification of autonomic disorders," in Autonomic Failure, eds C. J. Mathias and R. Bannister (Oxford: Oxford University Press), 3-9. doi: 10.1093/med/9780198566342.003. 0001

Benarroch, E. E. (2012). Postural tachycardia syndrome: a heterogeneous and multifactorial disorder. Mayo Clin. Proc. 87, 1214-1225. doi: 10.1016/j.mayocp. 2012.08.013

Bennaroch, E. E. (1993). The central autonomic network: functional organization, dysfunction, and perspective. Mayo Clin. Proc. 68, 988-1001. doi: 10.1016/ S0025-6196(12)62272-1

Bernhardt, B. C., Di Martino, A., Valk, S. L., and Wallace, G. L. (2016). Neuroimaging-based phenotyping of the autism spectrum. Curr. Top. Behav. Neurosci. 30, 341-355. doi: 10.1007/7854_2016_438

Boddaert, N., Chabane, N., Gervais, H., Good, C. D., Bourgeois, M., Plumet, M. H., et al. (2004). Superior temporal sulcus anatomical abnormalities in childhood autism: A voxel-based morphometry MRI study. NeuroImage 23, 364-369. doi: 10.1016/j.neuroimage.2004.06.016

Castori, M., Tinkle, B., Levy, H., Grahame, R., Malfait, F., and Hakim, A. (2017). A framework for the classification of joint hypermobility and related conditions. Am. J. Med. Genet. Part C Sem. Med. Genet. 175, 148-157. doi: 10.1002/ajmg.c. 31539

Dalton, K. M., Kalin, N. H., Grist, T. M., and Davidson, R. J. (2005). Neuralcardiac coupling in threat-evoked anxiety. J. Cogn. Neurosci. 17, 969-980. doi: 10.1162/0898929054021094

Duerden, E. G., Taylor, M. J., Soorya, L. V., Wang, T., Fan, J., and Anagnostou, E. (2013). Neural correlates of inhibition of socially relevant stimuli in adults with autism spectrum disorder. Brain Res. 1533, 80-90. doi: 10.1016/j.brainres.2013. 08.021

Ebisch, S. J. H., Gallese, V., Willems, R. M., Mantini, D., Groen, W. B., Romani, G. L., et al. (2011). Altered intrinsic functional connectivity of anterior and posterior insula regions in high-functioning participants with autism spectrum disorder. Human Brain Map. 32, 1013-1028. doi: 10.1002/hbm.2 1085

Eccles, J. A., Beacher, F., Gray, M. A., Jones, C. L., Minati, L., and Harrison, N. A. (2012). Brain structure and joint hypermobility: aelevance to the expression of psychiatric symptoms. Brit. J. Psychiatry 2012:92460. doi: 10.1192/bjp.bp.111. 092460

Eccles, J. A., Owens, A. P., Mathias, C. J., Umeda, S., and Critchley, H. D. (2015). Neurovisceral phenotypes in the expression of psychiatric symptoms. Front. Neurosci. 9:4. doi: 10.3389/fnins.2015.00004

Eccles, J., Lodice, V., Dowell, N., and Owens, A. (2014). Joint hypermobility and autonomic hyperactivity: relevance to neurodevelopmental disorders. J. Neurol. Neurosurg. Psychiatry 85:8883. doi: 10.1136/jnnp-2014-308 883.9

Evrard, H. C. (2019). The organization of the primate insular cortex. Front. Neuroanat. 13:43. doi: 10.3389/fnana.2019.00043

Freeman, R., Wieling, W., Axelrod, F. B., Benditt, D. G., Benarroch, E., Biaggioni, I., et al. (2011). Consensus statement on the definition of orthostatic hypotension, neurally mediated syncope and the postural tachycardia syndrome. Clin. Auton. Res. 21, 69-72.

Hadjikhani, N., Joseph, R. M., Manoach, D. S., Naik, P., Snyder, J., Dominick, K., et al. (2009). Body expressions of emotion do not trigger fear contagion in autism spectrum disorder. Soc. Cogn. Affect. Neurosci. 4, 70-78. doi: 10.1093/ scan/nsn038

Hutt, C., Hutt, S. J., Lee, D., and Ounsted, C. (1964). Arousal and childhood autism. Nature 204:908. doi: 10.1038/204908a0

Jänig, W., and Häbler, H. J. (2003). Neurophysiological analysis of targetrelated sympathetic pathways - From animal to human: Similarities and differences. Acta Physiol. Scand. 177, 255-274. doi: 10.1046/j.1365-201X.2003.0 1088.x

Kaufmann, H., Saadia, D., Polin, C., Hague, S., Singleton, A., and Singleton, A. (2003). Primary hyperhidrosis: Evidence for autosomal dominant inheritance. Clin. Auton. Res. 13, 96-98. doi: 10.1007/s10286-003-0082-x
Lai, F. C., Tu, Y. R., Li, Y. P., Li, X., Lin, M., Chen, J. F., et al. (2015). Nation wide epidemiological survey of primary palmar hyperhidrosis in the People's Republic of China. Clin. Auton. Res. 25, 105-108. doi: 10.1007/s10286-0140259-5

Martineau, J., Hernandez, N., Hiebel, L., Roche, L., Metzger, A., and BonnetBrilhault, F. (2011). Can pupil size and pupil responses during visual scanning contribute to the diagnosis of autism spectrum disorder in children? J. Psychiatric Res. 45, 1077-1082. doi: 10.1016/j.jpsychires.2011.0 1.008

Mathias, C. J., Deguchi, K., Bleasdale-Barr, K., and Kimber, J. R. (1998). Frequency of family history in vasovagal syncope. Lancet 352, 33-34. doi: 10.1016/S01406736(05)79513-1

Mathias, C. J., Low, D. A., Iodice, V., and Bannister, R. (2013). "Investigation of autonomic disorders," in Autonomic Failure, eds C. J. Mathias and R. Bannister (Oxford: Oxford University Press), 258-287. doi: 10.1093/med/9780198566342. 003.0024

Mathias, C. J., Low, D. A., Iodice, V., Owens, A. P., Kirbis, M., and Grahame, R. (2012). Postural tachycardia syndrome - Current experience and concepts. Nat. Rev. Neurol. 8, 22-34. doi: 10.1038/nrneurol.2011.187

Ming, X., Bain, J. M., Smith, D., and Brimacombe, M. (2011). Gold von-Simson G, Axelrod FB. Assessing autonomic dysfunction symptoms in children: A pilot study. J. Child Neurol. 26, 420-427. doi: 10.1177/088307381038 1921

Moraites, E., Vaughn, O. A., and Hill, S. (2014). Incidence and prevalence of hyperhidrosis. Dermatol. Clin. 32, 457-465. doi: 10.1016/j.det.2014.0 6.006

Ocon, A. J., Medow, M. S., Taneja, I., Clarke, D., and Stewart, J. M. (2009). Decreased upright cerebral blood flow and cerebral autoregulation in normocapnic postural tachycardia syndrome. Am. J. Physiol. Heart Circul. Physiol. 297, H664-H673. doi: 10.1152/ajpheart.00138. 2009

Owens, A. P., Low, D. A., Iodice, V., Critchley, H. D., and Mathias, C. J. (2017). The genesis and presentation of anxiety in disorders of autonomic overexcitation. Auton. Neurosci. Basic Clin. 203, 81-87. doi: 10.1016/j.autneu.2016.1 0.004

Owens, A. P., Low, D. A., Iodice, V., Mathias, C. J., and Critchley, H. D. (2016). Emotion and the autonomic nervous system-a two-way street: Insights from affective, autonomic and dissociative disorders. Curat. Ref. Collec. Neurosci. Biobehav. Psychol. 2016:1779. doi: 10.1016/B978-0-12-809324-5.01 799-5

Porges, S. W., Doussard-Roosevelt, J. A., Portales, A. L., and Greenspan, S. I. (1996). Infant regulation of the vagal "brake" predicts child behavior problems: a psychobiological model of social behavior. Dev. Psychobiol. 29, 69 7-712. doi: 10.1002/(SICI)1098-2302(199612)29:8\&lt;697::AID-DEV5\&gt;3.0. $\mathrm{CO} ; 2-\mathrm{O}$

Porges, S. W., Macellaio, M., Stanfill, S. D., McCue, K., Lewis, G. F., Harden, E. R., et al. (2013). Respiratory sinus arrhythmia and auditory processing in autism: Modifiable deficits of an integrated social engagement system? Int. J. Psychophysiol. 88, 261-270. doi: 10.1016/j.ijpsycho.2012.11.009

Quattrocki, E., and Friston, K. (2014). Autism, oxytocin and interoception. Neurosci. Biobehav. Rev. 47, 410-430. doi: 10.1016/j.neubiorev.2014.0 9.012

Sandroni, P., Novak, V., Opfer-Gehrking, T. L., Huck, C. A., and Low, P. A. (2000). Mechanisms of blood pressure alterations in response to the Valsalva maneuver in postural tachycardia syndrome. Clin. Auton. Res. 10, 1-5. doi: 10.1007/BF02291382

Santos, M., Uppal, N., Butti, C., Wicinski, B., Schmeidler, J., Giannakopoulos, P., et al. (2011). Von Economo neurons in autism: a stereologic study of the frontoinsular cortex in children. Brain Res. 1380, 206-217. doi: 10.1016/j. brainres.2010.08.067

Seeley, W. W., Merkle, F. T., Gaus, S. E., Craig, A. D., Allman, J. M., and Hof, P. R. (2012). Distinctive neurons of the anterior cingulate and frontoinsular cortex: A historical perspective. Cereb. Cortex 22, 245-250. doi: 10.1093/cercor/bh r005

Shetreat-Klein, M., Shinnar, S., and Rapin, I. (2014). Abnormalities of joint mobility and gait in children with autism spectrum disorders. Brain Dev. 36, 91-96. doi: 10.1016/j.braindev.2012.02.005 
Simms, M. L., Kemper, T. L., Timbie, C. M., Bauman, M. L., and Blatt, G. J. (2009). The anterior cingulate cortex in autism: heterogeneity of qualitative and quantitative cytoarchitectonic features suggests possible subgroups. Acta Neuropathol. 118, 673-684. doi: 10.1007/s00401-009-0568-2

Tinkle, B., Castori, M., Berglund, B., Cohen, H., Grahame, R., Kazkaz, H., et al. (2017). Hypermobile ehlers-danlos syndrome (a.k.a. ehlers-danlos syndrome Type III and Ehlers-Danlos syndrome hypermobility type): clinical description and natural history. Am. J. Med. Genet. Part C Sem. Med. Genet. 175, 48-69. doi: 10.1002/ajmg.c.31538

Toichi, M., and Kamio, Y. (2003). Paradoxical autonomic response to mental tasks in autism. J. Autism Dev. Disord. 33, 417-426. doi: 10.1023/A:102506281 2374

Watanabe, T., Yahata, N., Abe, O., Kuwabara, H., Inoue, H., Takano, Y., et al. (2012). Diminished medial prefrontal activity behind autistic social judgments of incongruent information. PLoS One 7:e39561. doi: 10.1371/journal.pone. 0039561
Conflict of Interest: The authors declare that the research was conducted in the absence of any commercial or financial relationships that could be construed as a potential conflict of interest.

Publisher's Note: All claims expressed in this article are solely those of the authors and do not necessarily represent those of their affiliated organizations, or those of the publisher, the editors and the reviewers. Any product that may be evaluated in this article, or claim that may be made by its manufacturer, is not guaranteed or endorsed by the publisher.

Copyright () 2021 Owens, Mathias and Iodice. This is an open-access article distributed under the terms of the Creative Commons Attribution License (CC BY). The use, distribution or reproduction in other forums is permitted, provided the original author(s) and the copyright owner(s) are credited and that the original publication in this journal is cited, in accordance with accepted academic practice. No use, distribution or reproduction is permitted which does not comply with these terms. 